\title{
Meson and baryon dispersion relations with Brillouin fermions
}

\author{
Stephan Dürr, ${ }^{1,2}$ Giannis Koutsou, ${ }^{3}$ and Thomas Lippert ${ }^{1,2}$ \\ ${ }^{1}$ Bergische Universität Wuppertal, Gaußstraße 20, 42119 Wuppertal, Germany \\ ${ }^{2}$ Jülich Supercomputing Center, Forschungszentrum Jülich, 52425 Jülich, Germany \\ ${ }^{3}$ Cyprus Institute, CaSToRC, 20 Kavafi Street, Nicosia 2121, Cyprus
}

(Received 17 September 2012; published 27 December 2012)

\begin{abstract}
We study the dispersion relations of mesons and baryons built from Brillouin quarks on one $N_{f}=2$ gauge ensemble provided by QCDSF. For quark masses up to the physical strange quark mass, there is hardly any improvement over the Wilson discretization, if either action is link-smeared and tree-level clover improved. For quark masses in the range of the physical charm quark mass, the Brillouin action still shows a perfect relativistic behavior, while the Wilson action induces severe cutoff effects. As an application we determine the masses of the $\Omega_{c}^{0}, \Omega_{c c}^{+}$and $\Omega_{c c c}^{++}$baryons on that ensemble.
\end{abstract}

DOI: 10.1103/PhysRevD.86.114514

PACS numbers: 12.38.Gc, 11.15.Ha, 14.20.Lq

\section{INTRODUCTION}

Wilson fermions offer an effective way of regulating the quark sector of QCD. Their conceptual simplicity entails a one-to-one correspondence between lattice and continuum flavor, a property which is particularly convenient for studying flavor physics in the standard model or in one of its extensions. The main disadvantage is that they induce cutoff effects in physical observables which are both parametrically and numerically large, i.e., $\propto a$, where $a$ is the lattice spacing, and the prefactor is sizable (for a discussion see, e.g., the recent review [1]).

Two technical remedies which have proven useful in mitigating the discretization effects are clover improvement and link smearing. The first one changes the parametric behavior to anything between $\alpha^{n} a$ and $a^{2}$ ( $\alpha$ is the strong coupling constant) [2], depending on whether the coefficient $c_{\mathrm{SW}}$ in Eq. (1) is adjusted in perturbation theory or nonperturbatively [3]. The second one concerns the links that enter the Dirac operator, in the covariant derivative and/or the clover term, and reduces the coefficient that multiplies the cutoff terms [4-8]. It turns out that either idea greatly enhances the other's effectiveness [9]; by combining a generic overall link smearing with an unsophisticated improvement strategy (e.g., the tree-level choice $\left.c_{\mathrm{SW}}=1\right)$, the amount of chiral symmetry breaking becomes as small as $a m_{\text {res }}=O\left(10^{-2}\right)[9,10]$.

In recent years such tree-level improved fat-link Wilson fermions have proven extremely successful, in particular, in enabling simulations of QCD with $N_{f}=2+1$ dynamical fermions directly at the physical mass point [11] (for an overview of the physics results obtained with such studies, see Ref. [1]). One can ask whether further action improvements would warrant the potential increase in CPU time needed to solve the Dirac equation $D x=b$ for a given right-hand side $b$. The goal of this paper is to investigate this question for the case of the "Brillouin fermion" proposed in Ref. [12]. The standard Wilson action (with clover improvement)

$$
\begin{aligned}
D^{\mathrm{Wil}}(x, y)= & \sum_{\mu} \gamma_{\mu} \nabla_{\mu}^{\mathrm{std}}(x, y)-\frac{1}{2} I \triangle^{\mathrm{std}}(x, y)+\frac{1}{2 \kappa} \delta_{x, y} \\
& -\frac{c_{\mathrm{SW}}}{2} \sum_{\mu<\nu} \sigma_{\mu \nu} F_{\mu \nu} \delta_{x, y}
\end{aligned}
$$

uses the simplest possible choice $\nabla_{\mu}^{\mathrm{std}}(x, y)=\left[U_{\mu}(x) \delta_{x+\hat{\mu}, y}\right.$ $\left.-U_{\mu}^{\dagger}(x-\hat{\mu}) \delta_{x-\hat{\mu}, y}\right] / 2$ of the symmetric covariant derivative and the simplest possible choice of the covariant Laplacian, $\triangle^{\text {std }}$, which is defined with the standard 9-point stencil. The Brillouin action [12]

$$
\begin{aligned}
D^{\mathrm{Bri}}(x, y)= & \sum_{\mu} \gamma_{\mu} \nabla_{\mu}^{\mathrm{iso}}(x, y)-\frac{1}{2} I \triangle^{\mathrm{bri}}(x, y)+\frac{1}{2 \kappa} \delta_{x, y} \\
& -\frac{c_{\mathrm{SW}}}{2} \sum_{\mu<\nu} \sigma_{\mu \nu} F_{\mu \nu} \delta_{x, y}
\end{aligned}
$$

uses the derivative $\nabla_{\mu}^{\text {iso }}$ and the Laplacian $\triangle^{\text {bri }}$, both of which have 81-point stencils chosen to minimize the amount of rotational symmetry breaking (in the transverse direction in case of $\nabla_{\mu}^{\text {iso }}$, overall in case of $\triangle{ }^{\text {bri }}$ ). The precise definition of these discretization schemes is given in the appendix of Ref. [12]. Incidentally, it turns out that Eq. (2) is an operator which is quite close to the one of Ref. [13], in spite of the construction being based on rather different principles.

The remainder of this article is organized as follows. In Sec. II we specify the set of $N_{f}=2$ gauge field configurations that we use to carry out our investigation, and we give further details of the link smearing and clover improvement (which we use both in the Wilson and in the Brillouin action, to compare like with like). Next, Sec. III contains the precise form of the Wuppertal smearing that we apply on both the source and the sink side of our propagators and describes the procedure by which we tune the mass parameters $\kappa$ in (1) and (2), to the correct value for the light, strange and charm quark mass. Section IV contains the central piece of our investigation, a comparison of the dispersion relation $E(\mathbf{p})^{2}$ as a function of the spatial momentum $\mathbf{p}^{2}$ for mesons and baryons built from Wilson and Brillouin fermions. As a 
phenomenological application, we compare in Sec. V the mass of the $\Omega_{c}^{0}$ baryon that we find to experiment, and we give the masses of the hitherto unobserved states $\Omega_{c c}^{+}$and $\Omega_{c c c}^{++}$on the ensemble considered. We summarize our findings in Sec. VI and arrange details of the baryon interpolating fields in an Appendix.

\section{ENSEMBLE AND VALENCE ACTION DETAILS}

The goal of our investigation is to compare the Wilson (1) and Brillouin (2) fermion actions in the valence sector, with special emphasis on the dispersion relation $E^{2}=E^{2}\left(\mathbf{p}^{2}\right)$ for mesons and baryons composed of such quarks. We shall use a freely available set of dynamical gauge field configurations, i.e., with the effect of light sea quark loops included. We select an ensemble out of the $N_{f}=2$ collection by QCDSF [14], namely, the one with

$\beta=5.29, \quad L / a=40, \quad T / a=64, \quad \kappa_{u d}^{\mathrm{sea}}=0.13632$

and $a M_{\pi}^{\text {sea }}=0.1034(8)$ [14]. Thus, $M_{\pi}^{\text {sea }} L=4.136$, which bears the promise of small finite-size effects. QCDSF determines the scale of their $\beta=5.29$ ensembles to be $a^{-1}=$ 2.71(2)(7) [15], tantamount to $a=0.0728(5)(19) \mathrm{fm}$, which implies $M_{\pi}^{\text {sea }} \simeq 280 \mathrm{MeV}$ and $L \simeq 2.9 \mathrm{fm}$.

Either action involves 3-fold APE smeared gauge links, where one smearing step in $d=4$ spacetime dimensions is given by

$$
\begin{aligned}
V_{\mu}(x)= & P_{\mathrm{SU}(3)}\left\{(1-\alpha) U_{\mu}(x)\right. \\
& \left.+\frac{\alpha}{2(d-1)} \sum_{ \pm \nu \neq \mu} U_{\nu}(x) U_{\mu}(x+\hat{\nu}) U_{\nu}^{\dagger}(x+\hat{\mu})\right\},
\end{aligned}
$$

and we use $\alpha_{4 \mathrm{D}}=0.72$. Here $P_{\mathrm{SU}(3)}$ denotes the back projection to $\mathrm{SU}(3)$ as described in Ref. [12], and $U$ is the unsmeared (original) gauge field. Note that these four-dimensionally smeared links enter both the (relevant) covariant derivative and the (irrelevant) clover term of the Wilson and Brillouin operators (for a summary of the options, see Ref. [9], but this choice is the simplest and, as far as we can see, the most effective one). In addition, the clover coefficient is set to its tree-level value $\left(c_{\mathrm{SW}}=1\right)$ for either action. In short, this is the same action as used in Ref. [12], except that we now use three steps of APE smearing rather than one.

\section{WUPPERTAL SMEARING AND QUARK MASS TUNING}

Wuppertal smearing amounts to the spreading of a vector $q(\mathbf{x}, t)$-with nontrivial support on the time slice $t$-within that time slice by means of $N_{\mathrm{W}}$ operations of the form [16]

$$
\begin{aligned}
q(\mathbf{x}, t) \rightarrow & \frac{\delta_{\mathbf{x}, \mathbf{y}}+\delta_{\mathrm{W}} \sum_{i}\left\{V_{i}(\mathbf{x}, t) \delta_{\mathbf{x}+\hat{i}, \mathbf{y}}+V_{i}^{\dagger}(\mathbf{x}-\hat{i}, t) \delta_{\mathbf{x}-\hat{i}, \mathbf{y}}\right\}}{1+6 \delta_{\mathrm{W}}} \\
& \times q(\mathbf{y}, t)
\end{aligned}
$$

with spreading parameter $\delta_{\mathrm{W}}$. The index $i$ runs over the three spatial directions, and an implicit summation over $\mathbf{y}$ takes place. Here the spatial links $V_{i}(\mathbf{x}, t)$ are to be generated with the three-dimensional version of the APE smearing (4) applied to the time slice $t$. After the Dirac equation $D p=q$ has been solved with the broadened source $q$, an identical spreading is applied to the solution $p$ (for each time slice separately), i.e., we use smearedsmeared propagators with the same smearing on the source and on the sink side.

In principle Wuppertal smearing has four parameters to adjust, namely, $\left(N_{3 \mathrm{D}}, \alpha_{3 \mathrm{D}}\right)$ of the three-dimensional APE smearing that the spatial links $V_{i}(\mathbf{x}, t)$ have undergone and $\left(N_{\mathrm{W}}, \delta_{\mathrm{W}}\right)$ in the recipe (5). For convenience we try to optimize the two pairs separately.

We first study the behavior of the spatial plaquette of the lattices (3) under repeated applications of the threedimensional version of the APE recipe (4). Our results are displayed in the left panel of Fig. 1. In Ref. [9] there is the perturbative stability bound $\alpha^{\mathrm{APE}}<(d-1) / d$ in $d$ spacetime dimensions. Our results suggest that any $\alpha_{3 \mathrm{D}}^{\mathrm{APE}}<$ $\alpha_{3 \mathrm{D}}^{\text {crit }}$ induces, asymptotically, a power-law falloff of $1-P_{3 \mathrm{D}}$ and thus allows us to drive the $3 \mathrm{D}$ plaquette arbitrarily small. In addition, $\alpha_{3 \mathrm{D}}^{\text {crit }}$ seems not too far from the perturbative prediction of $2 / 3$ [9]. Hence, our recommendation is to apply a large number of 3D APE smearings on the spatial links that enter the Wuppertal spreading (5), e.g., $\left(N_{3 \mathrm{D}}, \alpha_{3 \mathrm{D}}\right)=(300,0.6)$ or $(1000,0.6)$.

To decide on the second pair $\left(N_{\mathrm{W}}, \delta_{\mathrm{W}}\right)$ in Eq. (5) we first consider the root-mean-square radius of the smeared source as a function of these parameters, the former being defined through

$$
\left\langle r^{2}\right\rangle=\frac{\sum_{\mathbf{r}} \mathbf{r}^{2} q^{\dagger}(\mathbf{r}) q(\mathbf{r})}{\sum_{\mathbf{r}} q^{\dagger}(\mathbf{r}) q(\mathbf{r})} .
$$

Results from 5 configurations are shown in the right panel of Fig. 1. As expected, the contours look like hyperbolas; that is, the root-mean-square radius of $q$ is in the first place a function of the product $N_{\mathrm{W}} \delta_{\mathrm{W}}$. Next, we consider the effective mass of a meson made from $\kappa=0.112429$ Brillouin fermions (which is in the vicinity of $\kappa_{c}^{\text {Bri }}$, see below) for $\left(N_{\mathrm{W}}=10,30,90, \delta_{\mathrm{W}}=3\right)$, with results presented in Fig. 2. In this range a higher iteration count appears not to decrease the coupling to excited states, but rather seems to induce more noise in the correlators. As a consequence we decide to stay with the conservative parameter set $\left(N_{\mathrm{W}}=10, \delta_{\mathrm{W}}=0.5\right)$. 
To compare like with like, we wish to compare the dispersion relation for mesons and baryons put together from Wilson and Brillouin fermions at a fixed value of the light, strange or charm quark mass. This is most conveniently done by first tuning the two $\kappa$ values to get common values of $a M_{\pi}, a M_{s \bar{s}}$ and $a M_{c \bar{c}}$, respectively, for either discretization. For the mesons we use the $P P$ correlators, and given their cosh form it is advantageous to define the effective mass as

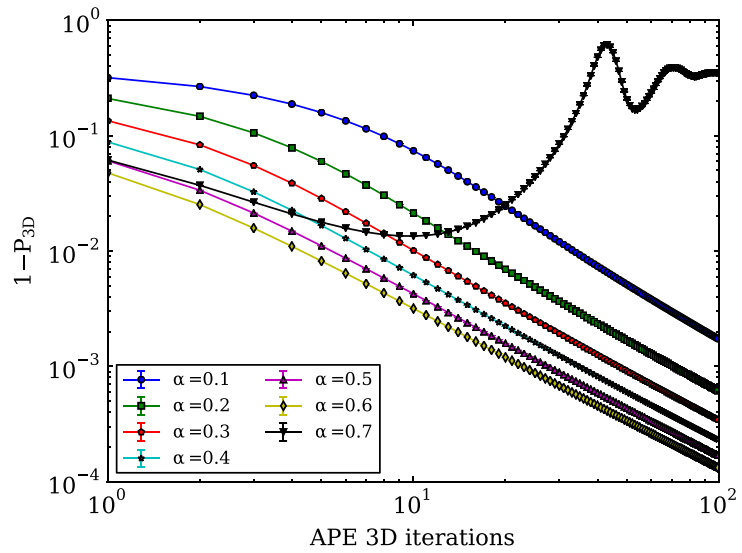

$a M_{\mathrm{eff}}(t)=\frac{1}{2} \log \left(\frac{C(t-1)+\sqrt{C(t-1)^{2}-C(T / 2)^{2}}}{C(t+1)+\sqrt{C(t+1)^{2}-C(T / 2)^{2}}}\right)$

because this modification remedies the falloff that the effective mass would show near the center of the box if the generic definition

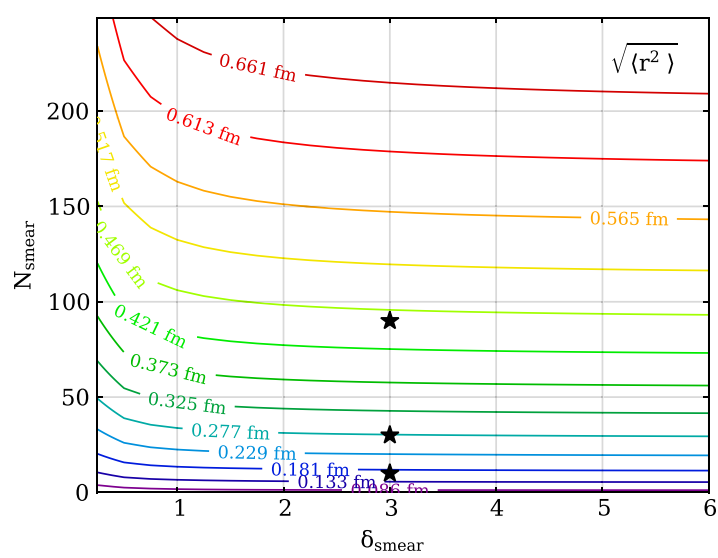

FIG. 1 (color online). Left: $1-\left\langle\operatorname{Re} \operatorname{Tr}\left(U_{\text {spat }}\right) / 3\right\rangle$ versus the number of 3D APE smearings for various values of $\alpha$; the perturbative bound is $\alpha_{3 \mathrm{D}}<2 / 3$ [9]. Right: contour plot of $\left\langle r^{2}\right\rangle^{1 / 2}$ of a Wuppertal smeared quark source; the asterisks refer to Fig. 2. Either plot is based on 5 configurations.
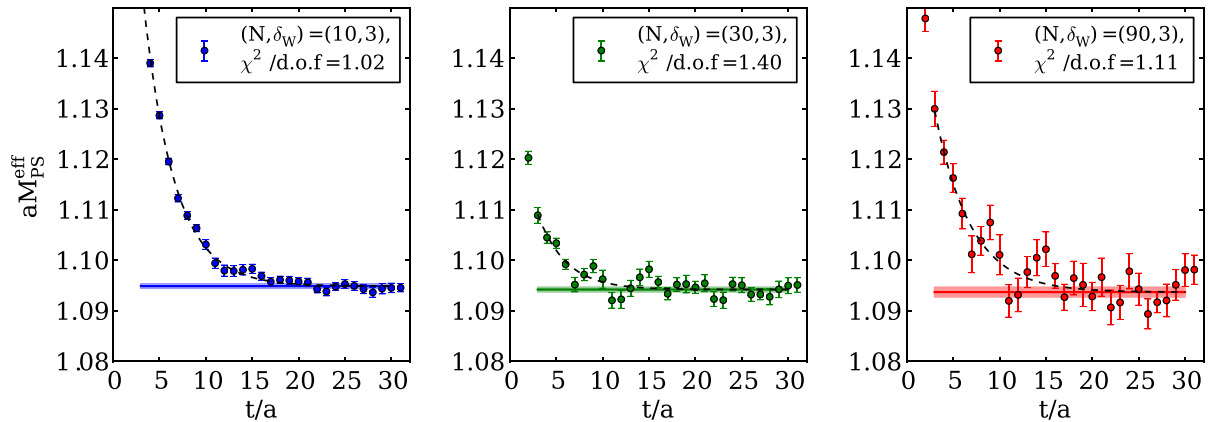

FIG. 2 (color online). Effective mass of the pseudoscalar $c \bar{c}$ meson made from Brillouin fermions with $\kappa=0.112429$ for three different widths (cf. Fig. 1) of the Wuppertal smeared sources and sinks.
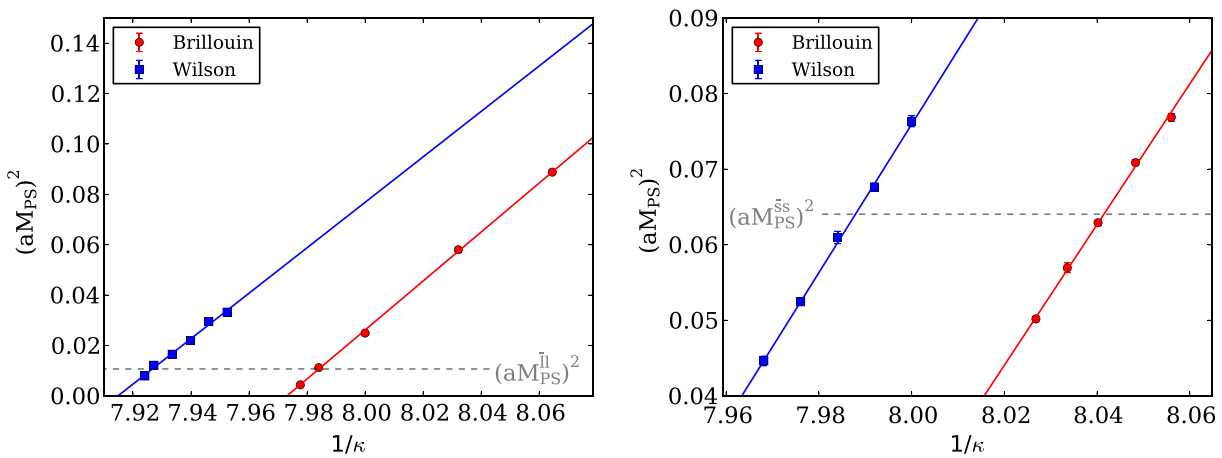

FIG. 3 (color online). Left: Tuning of $\kappa_{l}$ to achieve $\left(a M_{P}\right)^{2}=0.01069$ with either action. Right: Similar tuning of $\kappa_{s}$ to achieve $\left(a M_{P}\right)^{2}=0.06404$. 


$$
a M_{\mathrm{eff}}(t)=\frac{1}{2} \log \left(\frac{C(t-1)}{C(t+1)}\right)
$$

would be used. For baryons with (exact or approximate) projection to a definite parity (see Sec. IV below), we use the latter form, since these correlators do not show the cosh form.

For the light quark we demand the pion mass to be the same (in lattice units) as in the sea; that is $\left(a M_{P}\right)^{2}=$ 0.01069 , given the information provided beneath Eq. (3). We solve the Dirac equation for a few $\kappa$ values in the vicinity of the suspected target value and interpolate them linearly to obtain the desired $\kappa_{l}$, as shown in the left panel of Fig. 3. The results read

$$
\kappa_{l}^{\mathrm{Bri}}=0.125249, \quad \kappa_{l}^{\mathrm{Wil}}=0.126146 .
$$

For the strange quark we use the scale provided below Eq. (3) and the value $M_{s \bar{s}}=0.6858(7) \mathrm{GeV}$ which follows via $\sqrt{2 M_{K}^{2}-M_{\pi}^{2}}$ from the isospin-averaged and electromagnetically corrected masses $M_{\pi}=134$.8(3) $\mathrm{MeV}$ and $M_{K}=494.2(5) \mathrm{MeV}$ [17]. This gives the target value $\left(a M_{P}\right)^{2}=0.06404$, and a similar interpolation procedure, shown in the right panel of Fig. 3, yields

$$
\kappa_{s}^{\mathrm{Bri}}=0.1251902, \quad \kappa_{s}^{\text {Wil }}=0.1243560 .
$$

For the charm quark we proceed analogously to the strange case, except that we now use the value $M_{c \bar{c}}=$ 2.9810(11) GeV from PDG [18]. This yields the target value $\left(a M_{P}\right)^{2}=1.210$, and with essentially the same kind of procedure, we find

$$
\kappa_{c}^{\mathrm{Bri}}=0.112336, \quad \kappa_{c}^{\mathrm{Wil}}=0.112513 .
$$

A careful look at the left panel of Fig. 3 reveals that one of our trial $\kappa_{l}$ values for the Brillouin action happens to be rather light; for this point we find $a M_{\pi}=0.067(5)$, tantamount to $M_{\pi} \simeq 180 \mathrm{MeV}$. Following Refs. [11,19] we monitor the inverse iteration count of the solver (which is a proxy for the smallest eigenvalue of $D^{\dagger} D$ ) to make sure that we do not run into an "exceptional configuration" problem. The results of this monitoring are displayed in

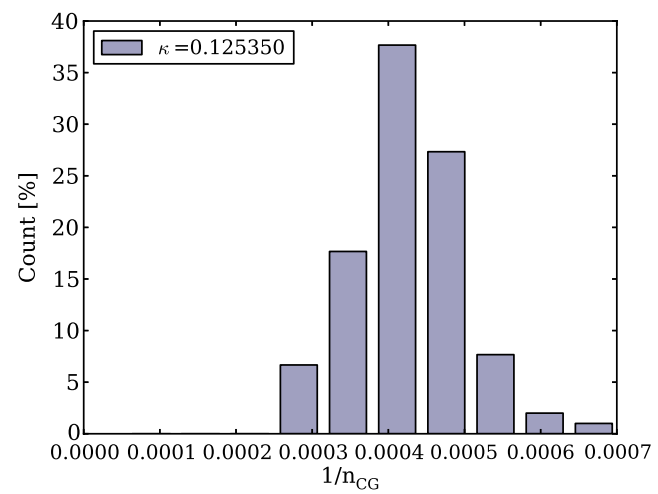

Fig. 4. Even for the lightest quark mass the distribution is roughly Gaussian, and the origin is 3 to 4 standard deviations away from the median. This shows that even in this strongly nonunitary regime (where $M_{\pi}^{\mathrm{val}}$ is about $100 \mathrm{MeV}$ lighter than $M_{\pi}^{\text {sea }}$ ), the Brillouin operator can be safely inverted. We consider this an encouraging sign of the great stability of our action against fluctuations of the small eigenmodes and think that this stability has the potential to render the Brillouin operator a cheap alternative to overlap or domain-wall fermions.

\section{MESON AND BARYON DISPERSION RELATIONS}

With the tuned $\kappa_{l}, \kappa_{s}, \kappa_{c}$ of Eqs. (9)-(11) in hand we are now in a position to study the dispersion relation $E^{2}=E^{2}\left(\mathbf{p}^{2}\right)$ for mesons and baryons composed of either Brillouin or Wilson fermions.

To this end we consider two-point correlators of the form

$$
C_{M}(t, \mathbf{p})=\sum_{\mathbf{x}}\left\langle J_{M}(\mathbf{x}, t) \bar{J}_{M}(\mathbf{0}, 0)\right\rangle e^{\mathbf{i p x}}
$$

$$
C_{\bar{B}}^{ \pm}(t, \mathbf{p})=\operatorname{Tr} \frac{1}{2}\left(1 \pm \gamma_{4}\right) \sum_{\mathbf{x}}\left\langle J_{B}(\mathbf{x}, t) \bar{J}_{B}(\mathbf{0}, 0)\right\rangle e^{\mathrm{i} \mathbf{p x}},
$$

where $M$ and $B$ define the quantum numbers of the meson or baryon, respectively. For nonzero momentum the correct parity projector in Eq. (13) would read $\frac{1}{2}\left(1 \pm \frac{E}{M} \gamma_{4}\right)[20,21]$, but we stay with the simpler form, since we are always interested in the lower-mass parity partner (which requires no projection at all). Finally, the sink and the source in Eq. (13) contain an uncontracted spinor index, say $\mu$ and $\nu$. The projection to a definite spin can be done with [22]

$$
\left(P^{3 / 2}\right)_{\mu \nu}=\delta_{\mu \nu}-\frac{1}{3} \gamma_{\mu} \gamma_{\nu}-\frac{1}{3 p^{2}}\left(\not p \gamma_{\mu} p_{\nu}-p_{\mu} \gamma_{\nu} \not p\right)
$$

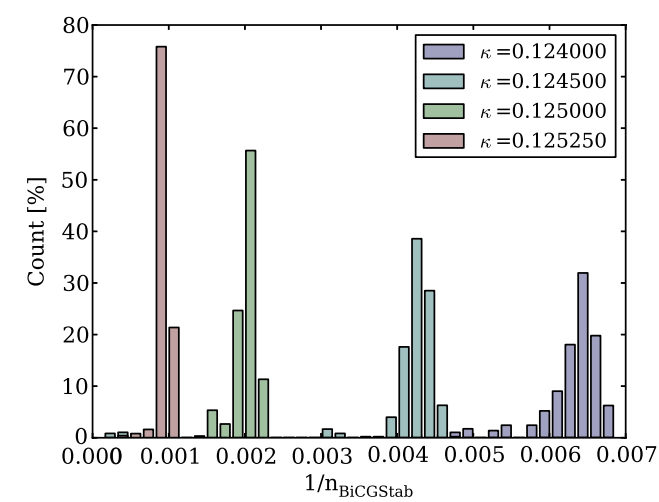

FIG. 4 (color online). Iteration count of the inversions with the trial $\kappa_{l}$ of Fig. 3 for the Brillouin action, using the CG (left) and the BiCGstab (right) algorithm for the lightest and all but the lightest masses, respectively. In each case $O(25)$ configurations are used. 
$\left(P_{11}^{1 / 2}\right)_{\mu \nu}=\frac{1}{3} \gamma_{\mu} \gamma_{\nu}-\frac{1}{p^{2}} p_{\mu} p_{\nu}+\frac{1}{3 p^{2}}\left(\not p \gamma_{\mu} p_{\nu}-p_{\mu} \gamma_{\nu} \not p\right)$

and similar expressions for $P_{12,21,22}^{1 / 2}$ as given in Eq. (9) of Ref. [22]. For $\mathbf{p}=0$ they simplify to

$$
\begin{gathered}
\left(P^{3 / 2}\right)_{i j}=\delta_{i j}-\frac{1}{3} \gamma_{i} \gamma_{j}, \\
\left(P_{11}^{1 / 2}\right)_{i j}=\frac{1}{3} \gamma_{i} \gamma_{j},
\end{gathered}
$$

which are easy to implement and good enough for many purposes (we are always interested in the lowest-mass state). For details of (more advanced) spin projection see Ref. [23].

Having defined the correlation functions, we can now move on to the dispersion relations. We consider spatial momenta $\mathbf{p}=2 \pi / L \cdot \mathbf{k}$ with $0 \leq \mathbf{k}^{2} \leq 20$. Thanks to the cubic symmetry among the spatial directions, in general, several $\mathbf{k}$ configurations contribute to a given $\mathbf{k}^{2}$. An effective mass plot before and after taking an average over the various contributions is shown in Fig. 5, for the pseudoscalar and vector meson, in the case of $\mathbf{k}^{2}=16$. We see no big discrepancies before the average is taken; hence, the averaging seems justified.

Repeating this procedure for all $\mathbf{k}^{2}$ yields the data presented in Fig. 6. We show the dispersion relations for the pseudoscalar and the vector meson with quark content $s \bar{s}$ (top), $s \bar{c}$ (middle) and $c \bar{c}$ (bottom). Evidently, for the heavier masses there is a significant difference between the Wilson data (red circles) and those with the Brillouin
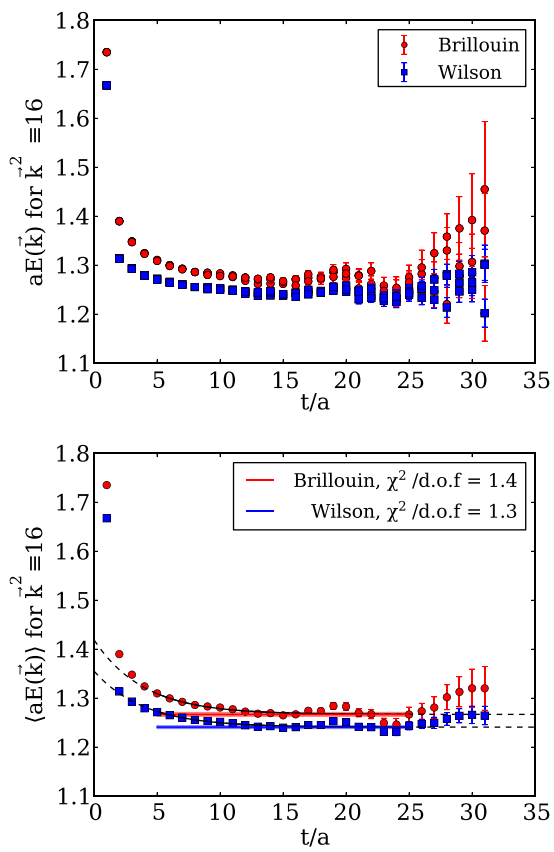

discretization (blue squares). The full black line is not a fit, but the relativistic dispersion relation $E^{2}=\mathbf{p}^{2}+M^{2}$, starting from the first data point (where the two actions were tuned to yield the same result for the $s \bar{s}$ and $c \bar{c}$ pseudoscalar states, but not for the remaining four states). This line shows that the discretization effects are induced by the Wilson action; within statistical errors the Brillouin data are free from such effects.

Similarly, we can work out the dispersion relations for baryons. The data for the "decuplet-type" states $\Omega_{\text {sss }}^{-}$, $\Omega_{c c c}^{++}$[which form a 20-plet under SU(4)], as well as for the "octet-type" states $\Omega_{c s s}^{0}, \Omega_{c c s}^{+}$[which form a $20^{\prime}$-plet under SU(4)] are shown in Fig. 7. To make it clear which states we consider, the interpolating fields of both the 20-plet and the 20'-plet are listed in the Appendix. Again, the full black line is not a fit but the relativistic dispersion relation $E^{2}=\mathbf{p}^{2}+M^{2}$, and we stress that no further tuning of $\kappa$ values was performed. Just like in the meson case we see no significant difference in the regime of the physical strange quark mass (which explains why we refrain from looking at even lighter $\kappa$ values, as such data would just be more noisy). However, with every strange quark that is replaced by a charm quark the difference becomes more pronounced, up to the point where the dispersion relation of the $\Omega_{c c c}^{++}$is seriously distorted with Wilson fermions, but relativistically correct with Brillouin fermions.

\section{MASSES OF MULTIPLY CHARMED BARYONS}

As a byproduct of our investigation, and with the goal of spurring further improvements, we can quote the masses of the $\Omega$ baryons considered in the previous section. Let us
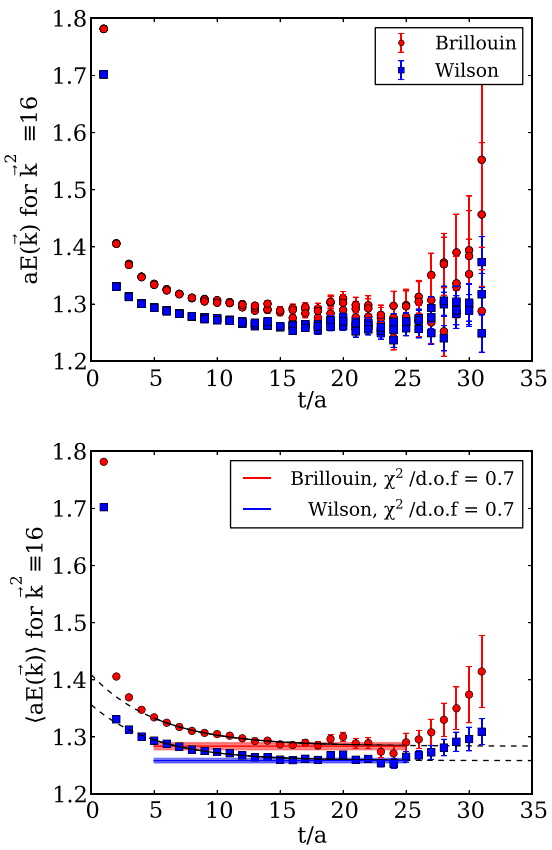

FIG. 5 (color online). Effective mass plots of the pseudoscalar (left) and vector (right) $c \bar{c}$ meson before (top) and after (bottom) averaging over the $\mathbf{k}$ configurations that contribute to $\mathbf{k}^{2}=16$. 

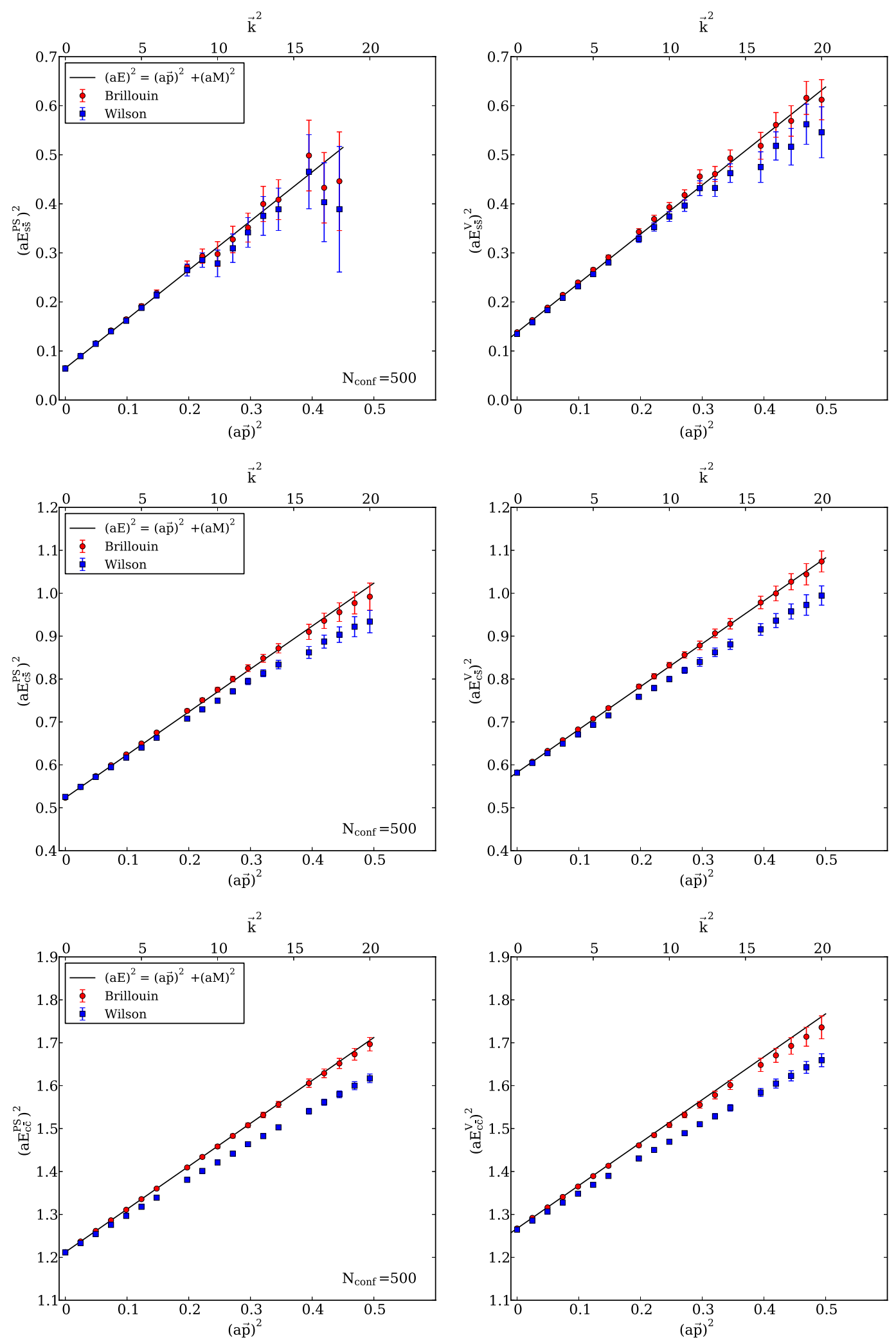

FIG. 6 (color online). Dispersion relations for the pseudoscalar (left) and vector (right) meson with $s \bar{s}$ (top), $s \bar{c}$ (middle), $c \bar{c}$ (bottom) quark content. The black line shows the relativistic $E^{2}=\mathbf{p}^{2}+M^{2}$. 

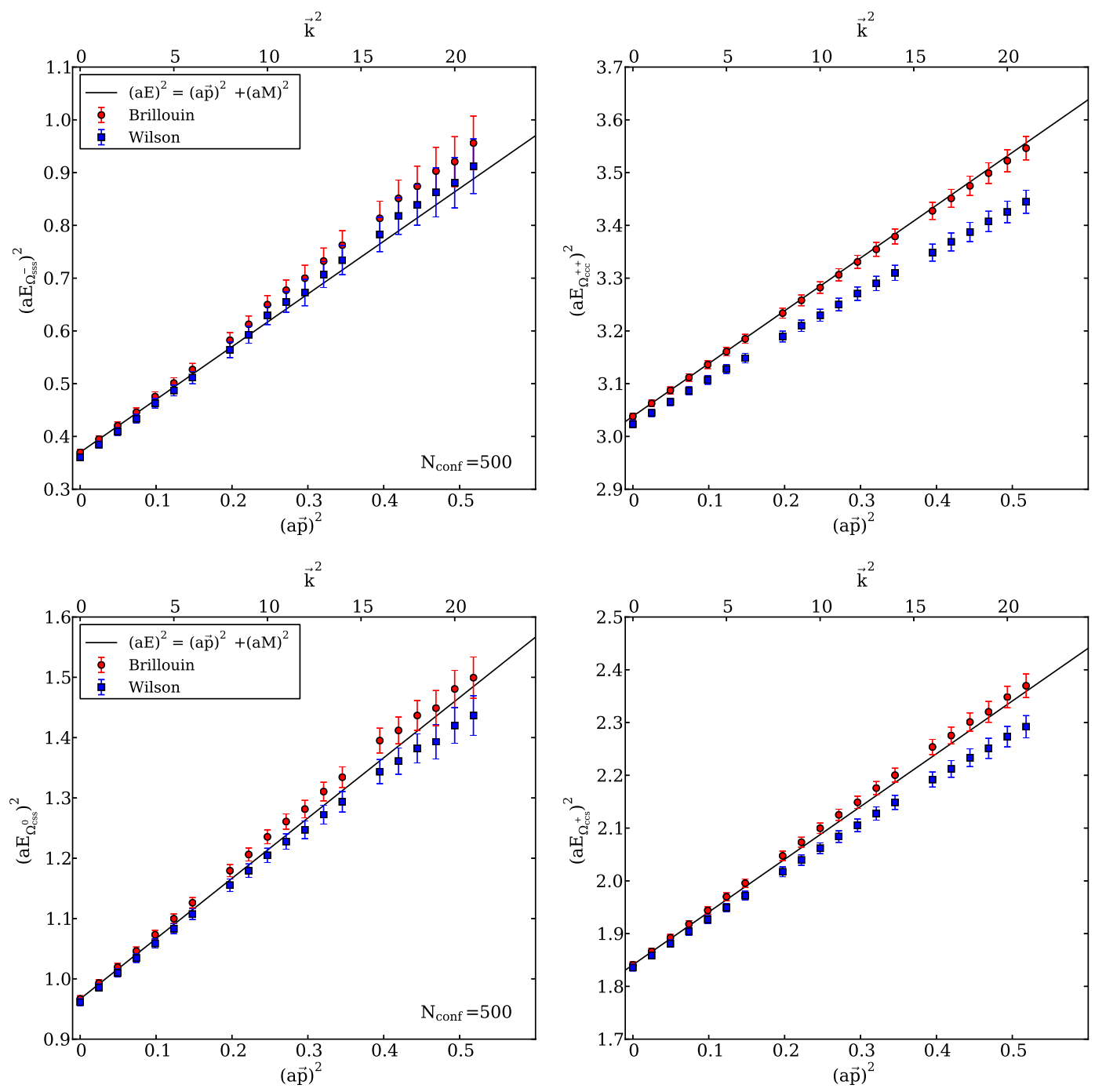

FIG. 7 (color online). Dispersion relations for the decuplet-type $\Omega_{s s s}^{-}, \Omega_{c c c}^{++}$(top left and right) and the octet-type $\Omega_{c s s}^{0}, \Omega_{c c s}^{+}$(bottom left and right). The black line shows the relativistic $E^{2}=\mathbf{p}^{2}+M^{2}$.
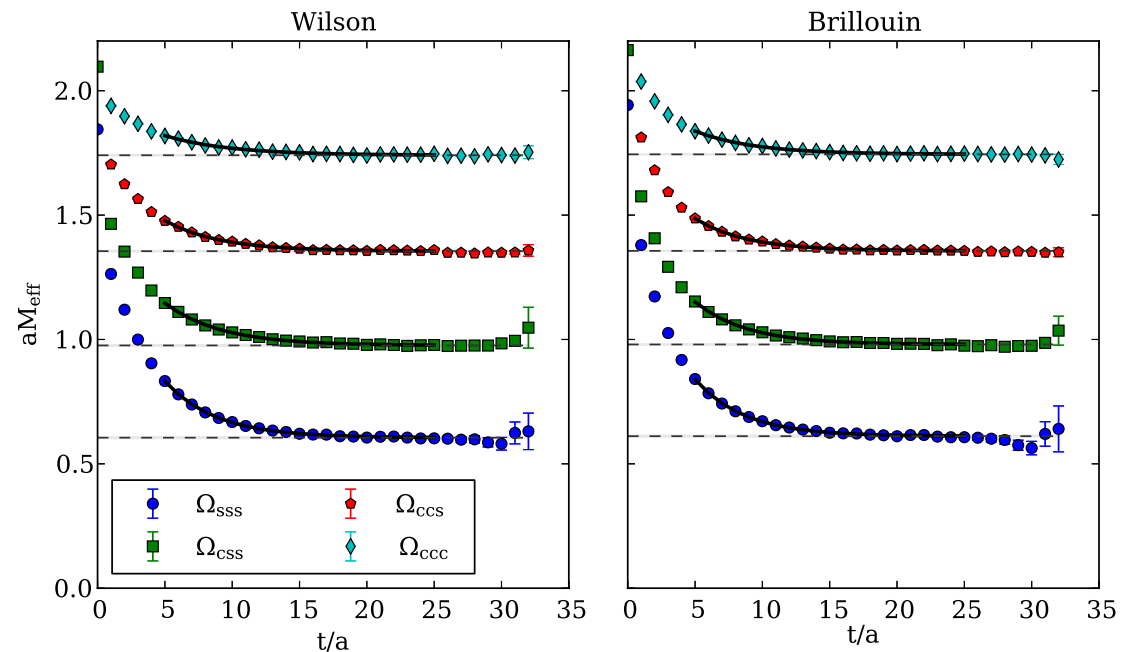

FIG. 8 (color online). Effective masses of the $\Omega_{s s s}, \Omega_{c c c}$ (decuplet-type states) and $\Omega_{s s c}, \Omega_{s c c}$ (octet-type states) at $\mathbf{p}=0$ for the Wilson (left) and Brillouin (right) action. 

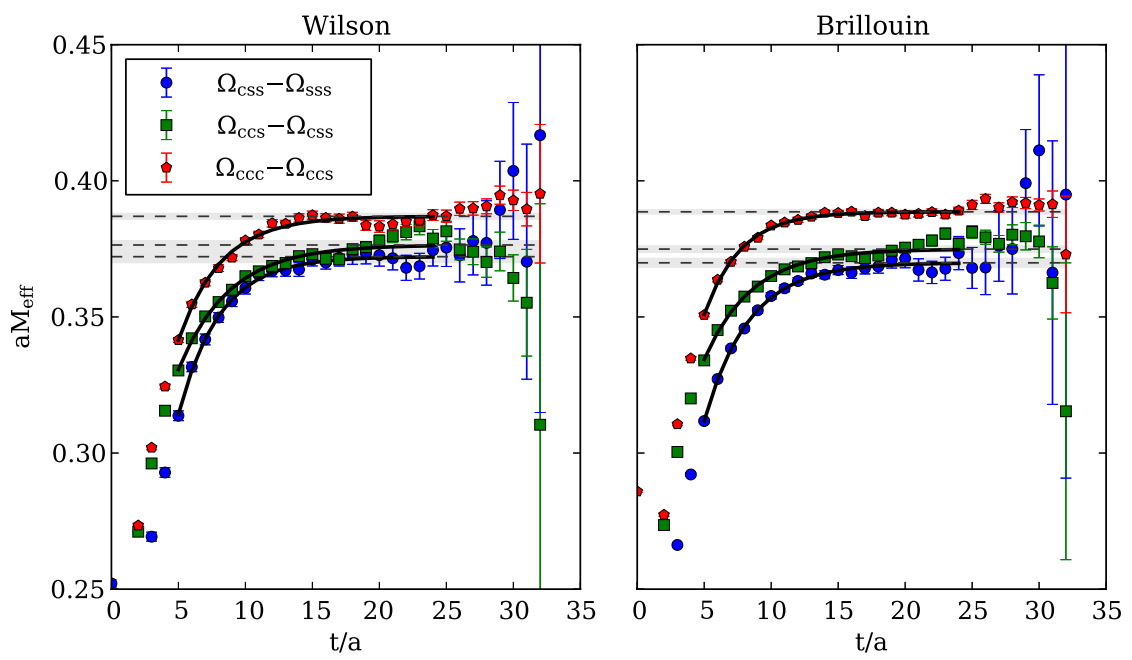

FIG. 9 (color online). Same as Fig. 8, but for the splittings $M_{\Omega_{c s s}}-M_{\Omega_{s s s}}$ and $M_{\Omega_{c c s}}-M_{\Omega_{c s s}}$ and $M_{\Omega_{c c c}}-M_{\Omega_{c c s}}$.

emphasize that this activity is based on the single ensemble (3). We will try to minimize the systematic effects on the numbers given below, but there is no way of reliably assessing the size of such systematic effects, given the data that we have.

Let us begin by showing the effective mass plots of the four states $\Omega_{s s s}^{-}, \Omega_{c s s}^{0}, \Omega_{c c s}^{+}, \Omega_{c c c}^{++}$in Fig. 8 . This is at $\mathbf{p}=0$, and we see no significant difference between the Wilson and the Brillouin action. Let us recall that the tuning was done in the meson sector, so this is a first nontrivial observation. To minimize the systematics it is usually a good idea to look at mass splittings and to form dimensionless ratios. The former trick mitigates the effect of excited states contaminations and possible finite volume effects, the latter one reduces the sensitivity to the overall scale. The effective mass plots for all adjacent mass splittings, based on ratios of correlators like $\left\langle C_{c s s}\right\rangle /\left\langle C_{s s s}\right\rangle$, are shown in Fig. 9. Even at this level of zoom, the quality of the data appears rather good, and we see only very mild differences between the Wilson and the Brillouin data. We list the relative mass splittings (normalized both with the lower one of the two states involved and with the $\Omega^{-}=\Omega_{\text {sss }}$ base state) in Table I.

Regarding phenomenological numbers we should first mention that the experimental mass of the $\Omega^{-}$(i.e., the $J^{P}=3 / 2^{+}, c=0, s=3$ state in Table III) is 1672.45 (29) $\mathrm{MeV}$, and the mass of the $\Omega_{c}^{0}$ (i.e., the $J^{P}=1 / 2^{+}$, $c=1, s=2$ state in Table II) is 2697.5(2.6) MeV [18]. Hence, with the ratios listed in Table I, we can only compare the mass of the $\Omega_{c}^{0}$ baryon to experiment, but not the masses of the $\Omega_{c c}^{+}$(i.e., the $J^{P}=1 / 2^{+}, c=2$, $s=1$ state in Table II) and of the $\Omega_{c c c}^{++}$(i.e., the $J^{P}=3 / 2^{+}, c=3, s=0$ state in Table III). We shall use the first three lines in Table I, taking the Brillouin number as our central value and the average difference to the Wilson number (0.007) as a uniform estimate of the systematic error. Adding all errors in quadrature yields

$$
\begin{gathered}
M_{\Omega_{c}^{0}}=2685(15) \mathrm{MeV} \\
M_{\Omega_{c c}^{+}}=3711(20) \mathrm{MeV} \\
M_{\Omega_{c c c}^{++}}=4774(24) \mathrm{MeV},
\end{gathered}
$$

and we emphasize that these errors do not include the effect of the (missing) limits $a \rightarrow 0$ and $M_{\pi}^{\text {sea }} \rightarrow$ 134.8 MeV. Nevertheless, Eq. (18) is consistent with the experiment, albeit with a large error. From these numbers it appears that the actual splitting is very close to equidistant, a notion which is also conveyed by Fig. 9.

We refrain from comparing our numbers to similar results in the recent literature on charm physics on the lattice [22,24-34]. We rather like to add that what is really called for, in our opinion, is a complete study with a reasonable assessment of all systematics involved, that is, with the continuum limit taken, with an interpolation or extrapolation to the physical values of $M_{\pi}$ and $M_{K}$ in the sea, and with an extrapolation to infinite box volume.

TABLE I. Relative mass splittings between the various $\Omega$ states, as determined with the Wilson (left) and the Brillouin (right) action. Here, the symbol $\Omega_{x y z}$ is meant as a shorthand for $M_{\Omega_{x y z}}$.

\begin{tabular}{lcc}
\hline \hline Combination & Wilson & Brillouin \\
\hline$\left(\Omega_{c s s}-\Omega_{s s s}\right) / \Omega_{s s s}$ & $0.615(6)$ & $0.605(5)$ \\
$\left(\Omega_{c c s}-\Omega_{c s s}\right) / \Omega_{s s s}$ & $0.622(5)$ & $0.613(4)$ \\
$\left(\Omega_{c c c}-\Omega_{c c s}\right) / \Omega_{s s s}$ & $0.639(4)$ & $0.636(4)$ \\
$\left(\Omega_{c c s}-\Omega_{c s s}\right) / \Omega_{c s s}$ & $0.386(2)$ & $0.383(2)$ \\
$\left(\Omega_{c c c}-\Omega_{c c s}\right) / \Omega_{c c s}$ & $0.286(1)$ & $0.287(1)$ \\
\hline \hline
\end{tabular}


TABLE II. Interpolating fields of SU(4) $20^{\prime}$-plet (octet-type) baryons with spin $1 / 2$. Throughout, the color indices are suppressed, i.e., $\boldsymbol{\epsilon}\left(x^{T} C \gamma_{5} y\right) z$ is to be read as $\epsilon_{a b c}\left(x_{a}^{T} C \gamma_{5} y_{b}\right) z_{c}$.

\begin{tabular}{|c|c|c|c|c|c|}
\hline Charm & Strange & Baryon & Interpolating field & $I$ & $I_{z}$ \\
\hline \multirow{8}{*}{$\bar{c}=0$} & $s=0$ & $p$ & $\epsilon\left(u^{T} C \gamma_{5} d\right) u$ & $1 / 2$ & $+1 / 2$ \\
\hline & & $n$ & $\epsilon\left(d^{T} C \gamma_{5} u\right) d$ & $1 / 2$ & $-1 / 2$ \\
\hline & $s=1$ & $\Sigma^{+}$ & $\epsilon\left(u^{T} C \gamma_{5} s\right) u$ & 1 & +1 \\
\hline & & $\Sigma^{0}$ & $\frac{1}{\sqrt{2}} \epsilon\left\{\left(u^{T} C \gamma_{5} s\right) d+\left(d^{T} C \gamma_{5} s\right) u\right\}$ & 1 & 0 \\
\hline & & $\Sigma^{-}$ & $\epsilon\left(d^{T} C \gamma_{5} s\right) d$ & 1 & -1 \\
\hline & $s=2$ & $\Xi^{0}$ & $\epsilon\left(s^{T} C \gamma_{5} u\right) s$ & $1 / 2$ & $+1 / 2$ \\
\hline & & $\Xi^{-}$ & $\epsilon\left(s^{T} C \gamma_{5} d\right) s$ & $1 / 2$ & $-1 / 2$ \\
\hline & $s=1$ & $\Lambda^{0}$ & $\frac{1}{\sqrt{6}} \epsilon\left\{2\left(u^{T} C \gamma_{5} d\right) s+\left(u^{T} C \gamma_{5} s\right) d-\left(d^{T} C \gamma_{5} s\right) u\right\}$ & 0 & 0 \\
\hline \multirow[t]{9}{*}{$c=1$} & $s=0$ & $\Sigma_{c}^{++}$ & $\epsilon\left(u^{T} C \gamma_{5} c\right) u$ & 1 & +1 \\
\hline & & $\Sigma_{c}^{+}$ & $\frac{1}{\sqrt{2}} \epsilon\left\{\left(u^{T} C \gamma_{5} c\right) d+\left(d^{T} C \gamma_{5} c\right) u\right\}$ & 1 & 0 \\
\hline & & $\Sigma_{c}^{0}$ & $\epsilon\left(d^{T} C \gamma_{5} c\right) d$ & 1 & -1 \\
\hline & $s=1$ & $\Xi_{c}^{\prime+}$ & $\frac{1}{\sqrt{2}} \epsilon\left\{\left(s^{T} C \gamma_{5} c\right) u+\left(u^{T} C \gamma_{5} c\right) s\right\}$ & $1 / 2$ & $+1 / 2$ \\
\hline & & $\Xi_{c}^{\prime 0}$ & $\frac{1}{\sqrt{2}} \epsilon\left\{\left(s^{T} C \gamma_{5} c\right) d+\left(d^{T} C \gamma_{5} c\right) s\right\}$ & $1 / 2$ & $-1 / 2$ \\
\hline & $s=2$ & $\Omega_{c}^{0}$ & $\epsilon\left(s^{T} C \gamma_{5} c\right) s$ & 0 & 0 \\
\hline & $s=0$ & $\Lambda_{c}^{+}$ & $\frac{1}{\sqrt{6}} \epsilon\left\{2\left(u^{T} C \gamma_{5} d\right) c+\left(u^{T} C \gamma_{5} c\right) d-\left(d^{T} C \gamma_{5} c\right) u\right\}$ & 0 & 0 \\
\hline & $s=1$ & $\Xi_{c}^{+}$ & $\frac{1}{\sqrt{6}} \epsilon\left\{2\left(s^{T} C \gamma_{5} u\right) c+\left(s^{T} C \gamma_{5} c\right) u-\left(u^{T} C \gamma_{5} c\right) s\right\}$ & $1 / 2$ & $+1 / 2$ \\
\hline & & $\Xi_{c}^{0}$ & $\frac{1}{\sqrt{6}} \epsilon\left\{2\left(s^{T} C \gamma_{5} d\right) c+\left(s^{T} C \gamma_{5} c\right) d-\left(d^{T} C \gamma_{5} c\right) s\right\}$ & $1 / 2$ & $-1 / 2$ \\
\hline \multirow[t]{3}{*}{$c=2$} & $s=0$ & $\Xi_{c c}^{++}$ & $\epsilon\left(c^{T} C \gamma_{5} u\right) c$ & $1 / 2$ & $+1 / 2$ \\
\hline & & $\Xi_{c c}^{+}$ & $\epsilon\left(c^{T} C \gamma_{5} d\right) c$ & $1 / 2$ & $-1 / 2$ \\
\hline & $s=1$ & $\Omega_{c c}^{+}$ & $\epsilon\left(c^{T} C \gamma_{5} s\right) c$ & 0 & 0 \\
\hline
\end{tabular}

TABLE III. Interpolating fields of SU(4) 20-plet (decuplet-type) baryons with spin 3/2. Throughout, the color indices are suppressed, i.e., $\epsilon\left(x^{T} C \gamma_{\mu} y\right) z$ is to be read as $\epsilon_{a b c}\left(x_{a}^{T} C \gamma_{\mu} y_{b}\right) z_{c}$.

\begin{tabular}{|c|c|c|c|c|c|}
\hline Charm & Strange & Baryon & Interpolating field & $I$ & $I_{z}$ \\
\hline \multirow[t]{10}{*}{$c=0$} & $s=0$ & $\Delta^{++}$ & $\epsilon\left(u^{T} C \gamma_{\mu} u\right) u$ & $3 / 2$ & $+3 / 2$ \\
\hline & & $\Delta^{+}$ & $\frac{1}{\sqrt{3}} \epsilon\left\{2\left(u^{T} C \gamma_{\mu} d\right) u+\left(u^{T} C \gamma_{\mu} u\right) d\right\}$ & $3 / 2$ & $+1 / 2$ \\
\hline & & $\Delta^{0}$ & $\frac{1}{\sqrt{3}} \epsilon\left\{2\left(d^{T} C \gamma_{\mu} u\right) d+\left(d^{T} C \gamma_{\mu} d\right) u\right\}$ & $3 / 2$ & $-1 / 2$ \\
\hline & & $\Delta^{-}$ & $\epsilon\left(d^{T} C \gamma_{\mu} d\right) d$ & $3 / 2$ & $-3 / 2$ \\
\hline & $s=1$ & $\Sigma^{\star+}$ & $\left.\frac{1}{\sqrt{3}} \epsilon\left\{2\left(u^{T} C \gamma_{\mu} s\right) u+u^{T} C \gamma_{\mu} u\right) s\right\}$ & 1 & +1 \\
\hline & & $\Sigma^{\star 0}$ & $\frac{1}{\sqrt{3}} \epsilon\left\{\left(u^{T} C \gamma_{\mu} d\right) s+\left(d^{T} C \gamma_{\mu} s\right) u+\left(s^{T} C \gamma_{\mu} u\right) d\right\}$ & 1 & 0 \\
\hline & & $\Sigma^{\star-}$ & $\left.\frac{1}{\sqrt{3}} \epsilon\left\{2\left(d^{T} C \gamma_{\mu} s\right) d+d^{T} C \gamma_{\mu} d\right) s\right\}$ & 1 & -1 \\
\hline & $s=2$ & $\Xi \star \Xi^{\star 0}$ & $\frac{1}{\sqrt{3}} \epsilon\left\{2\left(s^{T} C \gamma_{\mu} u\right) s+\left(s^{T} C \gamma_{\mu} s\right) u\right\}$ & $1 / 2$ & $+1 / 2$ \\
\hline & & $\Xi^{\star-}$ & $\frac{1}{\sqrt{3}} \epsilon\left\{2\left(s^{T} C \gamma_{\mu} d\right) s+\left(s^{T} C \gamma_{\mu} s\right) d\right\}$ & $1 / 2$ & $-1 / 2$ \\
\hline & $s=3$ & $\Omega^{-}$ & $\quad \epsilon\left(s^{T} C \gamma_{\mu} s\right) s$ & 0 & 0 \\
\hline \multirow[t]{6}{*}{$c=1$} & $s=0$ & $\Sigma_{c}^{\star++}$ & $\left.\frac{1}{\sqrt{3}} \epsilon\left\{2\left(u^{T} C \gamma_{\mu} c\right) u+u^{T} C \gamma_{\mu} u\right) c\right\}$ & 1 & +1 \\
\hline & & $\Sigma_{c}^{\star+}$ & $\frac{1}{\sqrt{3}} \epsilon\left\{\left(u^{T} C \gamma_{\mu} d\right) c+\left(d^{T} C \gamma_{\mu} c\right) u+\left(c^{T} C \gamma_{\mu} u\right) d\right\}$ & 1 & 0 \\
\hline & & $\Sigma_{c}^{\star 0}$ & $\left.\frac{1}{\sqrt{3}} \epsilon\left\{2\left(d^{T} C \gamma_{\mu} c\right) d+d^{T} C \gamma_{\mu} d\right) c\right\}$ & 1 & -1 \\
\hline & $s=1$ & $\Xi_{c}^{\star+}$ & $\frac{1}{\sqrt{3}} \epsilon\left\{\left(u^{T} C \gamma_{\mu} s\right) c+\left(s^{T} C \gamma_{\mu} c\right) u+\left(c^{T} C \gamma_{\mu} u\right) s\right\}$ & $1 / 2$ & $+1 / 2$ \\
\hline & & $\Xi_{c}^{\star 0}$ & $\frac{1}{\sqrt{3}} \epsilon\left\{\left(d^{T} C \gamma_{\mu} s\right) c+\left(s^{T} C \gamma_{\mu} c\right) d+\left(c^{T} C \gamma_{\mu} d\right) s\right\}$ & $1 / 2$ & $-1 / 2$ \\
\hline & $s=2$ & $\Omega_{c}^{\star 0}$ & $\frac{1}{\sqrt{3}} \epsilon\left\{2\left(s^{T} C \gamma_{\mu} c\right) s+\left(s^{T} C \gamma_{\mu} s\right) c\right\}$ & 0 & 0 \\
\hline \multirow[t]{3}{*}{$c=2$} & $s=0$ & $\Xi_{c c}^{\star++}$ & $\frac{1}{\sqrt{3}} \epsilon\left\{2\left(c^{T} C \gamma_{\mu} u\right) c+\left(c^{T} C \gamma_{\mu} c\right) u\right\}$ & $1 / 2$ & $+1 / 2$ \\
\hline & & $\Xi_{c c}^{\star+}$ & $\frac{1}{\sqrt{3}} \epsilon\left\{2\left(c^{T} C \gamma_{\mu} d\right) c+\left(c^{T} C \gamma_{\mu} c\right) d\right\}$ & $1 / 2$ & $-1 / 2$ \\
\hline & $s=1$ & $\Omega_{c c}^{\star+}$ & $\frac{1}{\sqrt{3}} \epsilon\left\{2\left(c^{T} C \gamma_{\mu} s\right) c+\left(c^{T} C \gamma_{\mu} c\right) s\right\}$ & 0 & 0 \\
\hline$c=3$ & $s=0$ & $\Omega_{c c c}^{++}$ & $\epsilon\left(c^{T} C \gamma_{\mu} c\right) c$ & 0 & 0 \\
\hline
\end{tabular}




\section{SUMMARY}

The goal of this work was to test whether a significant difference in meson and baryon dispersion relations is seen, depending on whether such a composite state is built from Wilson or Brillouin fermions. The main result is that for standard lattice spacings $\left(a^{-1}=2-3 \mathrm{GeV}\right)$ this is not the case if all quarks are, at most, as heavy as the physical strange quark, but significant differences become visible if one or several quarks are in the range of the physical charm quark mass-see Fig. 6 for mesons and Fig. 7 for baryons.

We should add that — even for heavy quarks - there is an alternative in case one is willing to give up on Lorentz invariance [35]. In the original version of this Fermilab method new anisotropy parameters were introduced which may be tuned to get the slope ("speed of light") of the pseudoscalar dispersion relation correct. Nowadays, it is more common to focus on the nonrelativistic behavior $E(\mathbf{p})=M_{1}+\mathbf{p}^{2} /\left(2 M_{2}\right)+\cdots$ and to choose $\kappa$ such that the kinetic mass $M_{2}$ is correct. In the first version the price to pay is the added expense, in terms of CPU and human time, to tune the parameters to sufficient precision (which is usually not too hard in a quenched context, but the issue will be aggravated by renormalization effects, once the charm quark is unquenched). In the second version only mass splittings among states with the same number of charm quarks may be considered, such that the rest mass $M_{1}$ drops out. By contrast the relativistic setup of the Brillouin action requires no tuning and no compromises to be made on the set of calculable observables; in our view it wins in terms of ease of use.

The Brillouin operator as proposed in Ref. [12] can be seen as a low-cost approximation to the concept of "perfect fermions" [36-39]. A recent development in the field of staggered fermions is to add a mixture of taste-S, V,T,A,P mass terms [40-44] such that the resulting action would have only one or two species in the continuum and an eigenvalue spectrum similar to the near-Ginsparg-Wilson spectrum of the Brillouin action (see, e.g., Fig. 22 of Ref. [12]).

In summary, we reach the conclusion that the added expense, in terms of CPU time, that the Brillouin action entails over the Wilson action is hardly justified if one is only interested in light quark spectroscopy (this may be different for structure functions). On the other hand, as soon as charm quarks are involved, the Brillouin action leads to a massive reduction of cutoff effects already in purely spectroscopic quantities. In particular, our Fig. 7 shows that the standard lore that $a M$ should not exceed 1 need not be true with Brillouin fermions; in this figure we see no cutoff effects in the range $(a E)^{2}=3.0-3.5$. All together, it seems our choice to use the Brillouin action to determine the quark mass ratio $m_{c} / m_{s}$ in Ref. [45] was justified, and we hope that this augurs well for the accuracy of our results (19) and (20).

\section{ACKNOWLEDGMENTS}

We thank Constantia Alexandrou and Zoltan Fodor for useful discussion. This work was supported in part by DFG through SFB TRR-55. The computing resources for this project were provided by Forschungszentrum Jülich $\mathrm{GmbH}$ through a VSR grant.

\section{APPENDIX: CHARMED BARYON INTERPOLATING FIELDS}

To avoid any confusion as to which states our numbers (18)-(20) would refer to, we give a list of the simplest baryon interpolators with charm and/or strangeness. We follow the naming convention of PDG [18] and group the operators according to their transformation properties under SU(4) in flavor space. Throughout, $C$ denotes the charge conjugation matrix, the transposition sign refers to spinor, and color indices are implicit, as described in the table captions.

Overall, the states separate into a $20^{\prime}$-plet of spin $1 / 2$ states, a 20-plet of spin 3/2 states, and a $\overline{4}$-plet under SU (4). These states are listed in Tables II, III, and IV, respectively.

The $20^{\prime}$-plet decomposes into the standard $c=0$ ground floor which transforms as an 8 under SU(3) [lines 1-8 in Table II], the $c=1$ first floor which decomposes into a 6 [lines 9-14] and a $\overline{3}$ [lines 15-17], and the $c=2$ second floor which transforms as a 3 under SU(3) [lines 18-20]. Regarding the $c=1$ level, it is worth noticing that the states of the 6 are symmetric under interchange of the two noncharmed quarks, whereas the states of the $\overline{3}$ are antisymmetric under this interchange. Here we adopt the rule that in the $20^{\prime}$-plet the diquark $\left(x^{T} C \gamma_{5} y\right)$ is antisymmetric under the interchange $x \leftrightarrow y$.

The structure of the 20-plet is somewhat simpler, since each fixed- $c$ floor has a unique transformation pattern under $\mathrm{SU}(3)$. It contains the standard $c=0$ ground floor which

TABLE IV. Interpolating fields of SU(4) $\overline{4}$-plet ("new-type") baryons. Throughout, the color indices are suppressed, i.e., $\epsilon\left(x^{T} C \gamma_{5} y\right) z$ is to be read as $\epsilon_{a b c}\left(x_{a}^{T} C \gamma_{5} y_{b}\right) z_{c}$.

\begin{tabular}{lccccc}
\hline \hline Charm & Strange & Baryon & Interpolating field & $I$ & $I_{z}$ \\
\hline$c=0$ & $s=1$ & $\Lambda^{\prime 0}$ & $\frac{1}{\sqrt{3}} \epsilon\left\{\left(u^{T} C \gamma_{5} d\right) s+\left(s^{T} C \gamma_{5} u\right) d+\left(d^{T} C \gamma_{5} s\right) u\right\}$ & 0 & 0 \\
$c=1$ & $s=0$ & $\Lambda_{c}^{\prime+}$ & $\frac{1}{\sqrt{3}} \epsilon\left\{\left(u^{T} C \gamma_{5} d\right) c+\left(c^{T} C \gamma_{5} u\right) d+\left(d^{T} C \gamma_{5} c\right) u\right\}$ & 0 & 0 \\
& $s=1$ & $\Xi_{c}^{\prime \prime+}$ & $\frac{1}{\sqrt{3}} \epsilon\left\{\left(u^{T} C \gamma_{5} s\right) c+\left(c^{T} C \gamma_{5} u\right) s+\left(s^{T} C \gamma_{5} c\right) u\right\}$ & $1 / 2$ & $+1 / 2$ \\
& & $\Xi_{c}^{\prime \prime 0}$ & $\frac{1}{\sqrt{3}} \epsilon\left\{\left(d^{T} C \gamma_{5} s\right) c+\left(c^{T} C \gamma_{5} d\right) s+\left(s^{T} C \gamma_{5} c\right) d\right\}$ & $1 / 2$ & $-1 / 2$ \\
\hline \hline
\end{tabular}


transforms as a 10 under SU(3) [lines 1-10 in Table III], the $c=1$ first floor which transforms as a 6 [lines 11-16], the $c=2$ second floor which transforms as a 3 [lines 17-19], and the $c=3$ one-point summit of the pyramid [line 20]. Here we adopt the rule that in the 20-plet the diquark $\left(x^{T} C \gamma_{\mu} y\right)$ is symmetric under the interchange $x \leftrightarrow y$.

The $\overline{4}$-plet decomposes into a $c=0$ ground floor which is an SU(3) singlet [line 1 in Table IV], and a $c=1$ first floor which transforms as a $\overline{3}$ [line 2-4]. In the former case the construction is based on the requirement that $\Sigma^{0} \propto$ $(u s) d+(d s) u, \Lambda^{0} \equiv \Lambda_{8}^{0} \propto 2(u d) s+(u s) d-(d s) u$, and $\Lambda^{\prime 0} \equiv \Lambda_{0}^{0} \propto(u d) s+(s u) d+(d s) u$ would be mutually orthogonal. In the latter case the interpolator is antisymmetric under the interchange of the two noncharmed quarks, if we adopt the rule that the diquark $\left(x^{T} C \gamma_{5} y\right)$ is antisymmetric under the interchange $x \leftrightarrow y$.
[1] Z. Fodor and C. Hoelbling, Rev. Mod. Phys. 84, 449 (2012).

[2] B. Sheikholeslami and R. Wohlert, Nucl. Phys. B259, 572 (1985).

[3] M. Luscher, S. Sint, R. Sommer, and P. Weisz, Nucl. Phys. B478, 365 (1996).

[4] T. A. DeGrand, A. Hasenfratz, and T. G. Kovacs (MILC Collaboration), Nucl. Phys. B547, 259 (1999).

[5] C. Bernard and T. DeGrand, Nucl. Phys. B, Proc. Suppl. 83-84, 845 (2000).

[6] M. Stephenson, C. DeTar, T.A. DeGrand, and A. Hasenfratz, Phys. Rev. D 63, 034501 (2000).

[7] J. M. Zanotti, S. Bilson-Thompson, F. Bonnet, P. Coddington, D. Leinweber, A. Williams, J. Zhang, W. Melnitchouk, and F. Lee (CSSM Collaboration), Phys. Rev. D 65, 074507 (2002).

[8] T. DeGrand, A. Hasenfratz, and T. G. Kovacs, Phys. Rev. D 67, 054501 (2003).

[9] S. Capitani, S. Durr, and C. Hoelbling, J. High Energy Phys. 11 (2006) 028.

[10] S. Durr, Comput. Phys. Commun. 180, 1338 (2009).

[11] S. Durr, Z. Fodor, C. Hoelbling, S. D. Katz, S. Krieg, T. Kurth, L. Lellouch, T. Lippert, K. K. Szabó, and G. Vulvert, J. High Energy Phys. 08 (2011) 148.

[12] S. Durr and G. Koutsou, Phys. Rev. D 83, 114512 (2011).

[13] W. Bietenholz and I. Hip, Nucl. Phys. B570, 423 (2000).

[14] W. Bietenholz, M. Gockeler, R. Horsley, Y. Nakamura, D. Pleiter, P.E. L. Rakow, G. Schierholz, and J. M. Zanotti, Phys. Lett. B 687, 410 (2010).

[15] G. S. Bali et al. (QCDSF Collaboration), Phys. Rev. Lett. 108, 222001 (2012).

[16] S. Gusken, U. Low, K. H. Mutter, R. Sommer, A. Patel, and K. Schilling, Phys. Lett. B 227, 266 (1989).

[17] G. Colangelo et al. (FLAG), Eur. Phys. J. C 71, 1695 (2011).

[18] K. Nakamura et al. (Particle Data Group), J. Phys. G 37, 075021 (2010).

[19] L. Del Debbio, L. Giusti, M. Luscher, R. Petronzio, and N. Tantalo, J. High Energy Phys. 02 (2006) 011.

[20] F. X. Lee and D. B. Leinweber, Nucl. Phys. B, Proc. Suppl. 73, 258 (1999).

[21] D. B. Leinweber, W. Melnitchouk, D. G. Richards, A. G. Williams, and J. M. Zanotti, Lect. Notes Phys. 663, 71 (2005).

[22] K. C. Bowler, R. D. Kenway, O. Oliveira, D. G. Richards, P. Ueberholz, L. Lellouch, J. Nieves, C. T. Sachrajda, N. Stella, and H. Wittig (UKQCD Collaboration), Phys. Rev. D 54, 3619 (1996).
[23] R. G. Edwards, J. J. Dudek, D. G. Richards, and S. J. Wallace, Phys. Rev. D 84, 074508 (2011).

[24] R. Lewis, N. Mathur, and R. M. Woloshyn, Phys. Rev. D 64, 094509 (2001).

[25] N. Mathur, R. Lewis, and R. M. Woloshyn, Phys. Rev. D 66, 014502 (2002).

[26] J. M. Flynn, F. Mescia, and A.S. Bin Tariq (UKQCD Collaboration), J. High Energy Phys. 07 (2003) 066.

[27] H. Na and S. Gottlieb, Proc. Sci. LATTICE 2008 (2008) 119.

[28] L. Liu, H.-W. Lin, K. Orginos, and A. Walker-Loud, Phys. Rev. D 81, 094505 (2010).

[29] H.-W. Lin, S. D. Cohen, L. Liu, N. Mathur, K. Orginos, and A. Walker-Loud, Comput. Phys. Commun. 182, 24 (2011).

[30] D. Mohler and R. M. Woloshyn, Phys. Rev. D 84, 054505 (2011).

[31] Y. Namekawa et al. (PACS-CS Collaboation), Phys. Rev. D 84, 074505 (2011).

[32] H.-W. Lin, Chin. J. Phys. (Taipei) 49, 827 (2011).

[33] C. Alexandrou, J. Carbonell, D. Christaras, V. Drach, M. Gravina, and M. Papinutto, arXiv:1205.6856.

[34] R. A. Briceno, H.-W. Lin, and D. R. Bolton, Phys. Rev. D 86, 094504 (2012).

[35] A.X. El-Khadra, A.S. Kronfeld, and P. B. Mackenzie, Phys. Rev. D 55, 3933 (1997).

[36] P. Hasenfratz and F. Niedermayer, Nucl. Phys. B414, 785 (1994).

[37] W. Bietenholz and U.J. Wiese, Nucl. Phys. B464, 319 (1996).

[38] P. Hasenfratz, S. Hauswirth, K. Holland, T. Jorg, F. Niedermayer, and U. Wenger, Int. J. Mod. Phys. C 12, 691 (2001).

[39] P. Hasenfratz, S. Hauswirth, T. Jorg, F. Niedermayer, and K. Holland, Nucl. Phys. B643, 280 (2002).

[40] M.F. L. Golterman and J. Smit, Nucl. Phys. B245, 61 (1984).

[41] D. H. Adams, Phys. Lett. B 699, 394 (2011).

[42] C. Hoelbling, Phys. Lett. B 696, 422 (2011).

[43] M. Creutz, T. Kimura, and T. Misumi, J. High Energy Phys. 12 (2010) 041.

[44] P. de Forcrand, A. Kurkela, and M. Panero, J. High Energy Phys. 04 (2012) 142.

[45] S. Durr and G. Koutsou, Phys. Rev. Lett. 108, 122003 (2012). 